\title{
Intervenciones comunitarias en la educación formal: el desafío de garantizar la cohesión en la diversidad 1
}

\author{
Community interventions in formal education: \\ the challenge of ensuring cohesion in diversity
}

\section{Mauricio David Fuentes Simonini,* Leticia Gómez Guerrero** y Natalia Porcelli Llano***}

\footnotetext{
* Licenciado en Ciencias de la Educación (Universidad de Buenos Aires), con cursos de posgrado vinculados a educación en contextos de vulnerabilidad social y a la investigación educativa. Cursa la Maestría en Psicología y Educación en la Facultad de Psicología (UdelaR). Profesor en el Colegio Santa María. Director ejecutivo de la Asociación Civil Obsur. Investigador de la Agencia Nacional de Investigación e Innovación (ANII) en el marco del Fondo Sectorial de Educación (FSEd).
}

$\bowtie \cdot$ mauriciof1982@gmail.com https://orcid.org/0000-0002-9584-8770

** Maestra (Instituto de Formación Docente, Fray Bentos). Estudiante de la Licenciatura en Ciencias Antropológicas de la UdelaR. Maestra en el Colegio Santa María. Investigadora FSEd-ANII.

?]?galsorgo@gmail.com https://orcid.org/0000-0002-9994-6001

*** Maestra (Institutos Normales, Montevideo). Estudiante de la Licenciatura en Trabajo Social (UdelaR). Maestra referente en el CAIF Padre Víctor (Cerrito, Montevideo). Investigadora FSEd-ANII.

$\triangle$ pporcellinatalia@gmail.com

https://orcid.org/0000-0002-0258-9221

\section{RECIBIDO: 4.9 .2019}

ACEPTADO: 16.11 .2019

1 La investigación de la que se desprende el presente artículo se desarrolla en el marco del Fondo Sectorial de Educación "Investigación desde la perspectiva de los educadores sobre sus prácticas educativas", de la Agencia Nacional de Investigación e Innovación (ANII), con el apoyo del Ministerio de Educación y Cultura (MEC) y la Administración Nacional de Educación Pública (ANEP). 


\section{Resumen}

El siguiente artículo presenta un avance de una investigación desarrollada en 24 instituciones públicas de educación formal en la ciudad de Montevideo. En el marco de dicha investigación, se describen las intervenciones que diversos actores comunitarios impulsan en escuelas ubicadas en contexto de vulnerabilidad social, dando cuenta de cuáles son esos actores y cuáles las dimensiones de la vida institucional en que dichas intervenciones tienen impacto. Esta descripción revela la gran heterogeneidad del conjunto de intervenciones, a la vez que presenta el desafío de la permeabilidad selectiva que los maestros directores deben instrumentar como forma de garantizar la cohesión en la diversidad.

Palabras clave: educación formal, grupo desfavorecido, investigación pedagógica.

\section{Abstract}

The following article presents an advancement of a research developed in 24 public institutions of formal education in the city of Montevideo. Within the framework of this research, the interventions that various community actors promote in schools located in contexts of social vulnerability are described, identifying these actors as well as the dimensions of institutional life in which these interventions have an impact. This description reveals the great variety of intervention sets displayed, as well as the challenges of implementing selective permeability that teacher principals must face in order to guarantee cohesion in diversity.

Keywords: formal education, disadvantaged groups, educational research.

\section{Relaciones entre la escuela y la comunidad}

El término comunidad pareciera haber cobrado auge en las investigaciones sociales contemporáneas, especialmente para hacer énfasis en su dimensión instituyente y en la posibilidad que muestra la acción comunitaria como modalidad alternativa de funcionamiento del sistema social (Zibechi, 2006). Sin embargo, es importante tener en cuenta que, si bien dicho concepto evoca un conjunto de elementos vinculados con las relaciones humanas cercanas y horizontales, la «imagen unitaria y esencialista de comunidad invisibiliza las diferencias, tensiones y conflictos propios de todo colectivo o entidad social» (Torres, 2013, p. 12).

Las ideas clásicas de comunidad pueden encontrarse en varias corrientes sociológicas de la modernidad. Así, por ejemplo, desde la Escuela de Chicago (Torres, 2013) se hace hincapié en los aspectos relacionales y se concibe a la comunidad como un grupo de personas que viven de manera interdependiente y con arraigo territorial. Durkheim 
encuentra en la comunidad la base de conciencia colectiva para el desarrollo de creencias y sentimientos comunes: lo comunitario se presenta como un fenómeno que permite la convivencia desde una perspectiva de desarrollo ético y político.

Sin embargo, la experiencia actual de los territorios en contextos de vulnerabilidad social subraya que este proceso de reciprocidad e interdependencia inherente a lo comunitario sucede en el marco de tensiones.

La noción de vulnerabilidad social es fundamental para comprender la realidad que atraviesan algunos territorios específicos de las ciudades latinoamericanas, y particularmente de Montevideo. Robert Castel (2009) menciona que existe una relación directa entre el lugar que ocupan los individuos en la estructura de las relaciones laborales y los diferentes dispositivos de protección social que se desarrollan en la sociedad. Las modalidades de protección a las que acceden los individuos respecto a su lugar en la estructura de relaciones laborales pueden ser denominadas zonas de cohesión social.

La asociación trabajo estable e inserción relacional sólida caracteriza una zona de integración. A la inversa, la ausencia de participación en alguna actividad productiva y el aislamiento relacional conjugan sus efectos negativos para producir la exclusión, o más bien, como trataré de demostrarlo, la desafiliación. La vulnerabilidad social es una zona intermedia, inestable, que conjuga la precariedad del trabajo y la fragilidad de los soportes de proximidad. (Castel, 2009, p. 15)

En diversos barrios de Montevideo los indicadores dan cuenta de esta conjunción entre la precariedad en el acceso al mercado laboral y la dificultad para acceder a los sistemas de protección social. Sin embargo, es interesante ver como este fenómeno no necesariamente implica homogeneidad en la población de estos barrios.

La emergencia de asentamientos a lo largo de las últimas décadas del siglo XX, consecuencia de fenómenos migratorios internos y de segregación residencial tradicional, se ve complementada por un fenómeno al que se puede denominar segregación acaIlada (Carman, Vieira da Cunha y Segura, 2013). Esto implica que existe una nueva modalidad de segregación que surge como consecuencia de un conjunto de políticas que, en apariencia, tienen una finalidad positiva.

El resultado de este proceso es que los barrios en los que se suceden las interacciones cotidianas de los individuos ya no pueden ser comprendidos presumiendo su homogeneidad. En este nuevo escenario, «un territorio popular no es una unidad social en la que conviven armoniosamente sus habitantes y que comparten uniformes ideas, valores y propósitos. En estos territorios coexiste una pluralidad de grupos humanos, con intereses diferentes y muchas veces contrapuestos» (Torres, 2013, p. 165).

Dentro de este marco de contradicciones y fuerzas contrapuestas encontramos las instituciones de educación formal, en especial las escuelas. La escuela, como institución 
educativa, se encuentra en una relación cada vez más constante con la comunidad a la que pertenece, y aparece más permeable a los vínculos con las personas e instituciones que forman parte de dicha comunidad. «Las familias, las entidades locales, los grupos sociales deben preocuparse de que los niños puedan llevar a cabo experiencias significativas en contacto directo con todos los integrantes de la vida organizada que tengan algo que ofrecer a la educación» (Alfieri, 1999, p. 177).

En Uruguay, la normativa vigente establece un conjunto de modalidades a partir de las cuales la comunidad puede formar parte de los procesos que acontecen en el marco de las instituciones educativas. La ley 18437 (Ley General de Educación) prevé en su artículo 41 que los centros educativos en los que se desarrollen tareas de enseñanza pueden celebrar convenios con otras instituciones dentro de su función específica. Estos acuerdos, en función de lo que emana de dicha norma, pueden implicar aspectos vinculados tanto a su misión como a las cuestiones de gestión o presupuesto. A su vez, los artículos 48 y 53 hacen referencia a la importancia que adquiere la participación de la sociedad y de las personas externas a la institución educativa en diversos procesos que ocurren dentro de esta, tanto en lo que refiere a aspectos operativos como en los procesos de toma de decisiones. Finalmente, el capítulo IX establece los derechos y responsabilidades de los padres y madres de alumnos, y explicita los mecanismos de funcionamiento de los consejos de participación (ley 18437, 2009).

La investigación educativa en Uruguay ha dado cuenta de las relaciones entre escuela y comunidad. Pereda $(2013,2018)$, Alonso y Viscardi (2013) y Echevarriarza, Pereda y Silveira (2011) son algunos antecedentes relevantes que ponen en evidencia diferentes modalidades en las que la institución educativa se vincula con la comunidad en la que se inserta.

Esta apertura en los vínculos cada vez más frecuentes entre la comunidad y la institución educativa lleva a la posibilidad de que en esta última se sucedan cada vez más intervenciones llevadas adelante por actores de la comunidad.

Una intervención puede entenderse como «una relación transformadora de una situación en la cual agentes externos (a la comunidad) y agentes internos (de la comunidad), conjugan esfuerzos para solucionar algún problema, atender a alguna carencia o cumplir una expectativa» (Montero Rivas, 2012, p. 69).

Es claro, entonces, que en las instituciones educativas en general, y en las escuelas en particular, la participación de la comunidad se encuentra habilitada por la normativa vigente. En algunos casos esa participación adquiere carácter de intervención, en la medida en que se transforma en una práctica que busca modificar determinado problema de la realidad. Cabe destacar que en los contextos de vulnerabilidad social estas intervenciones son llevadas adelante por actores que pertenecen a ese conjunto heterogéneo que se denomina comunidad. 


\section{Intervenciones comunitarias: un relevamiento descriptivo}

Las intervenciones comunitarias tienen como fin dar respuesta a una problemática, carencia o necesidad. En este sentido, es posible reconocer que, al intervenir en las escuelas, los actores comunitarios procuran dar respuesta a las dificultades que encuentran, tanto en la función institucional como en las condiciones para llevarla a cabo.

La ubicación en el espacio geográfico mostrará, por un lado, el grado de conexión o aislamiento del establecimiento y su entorno respecto de los centros en los que se concentra el poder social e institucional. Por otro, permitirá comprender su posible significación para el tipo de población que atiende y las condiciones particulares de trabajo que esa población crea a la escuela. También mostrará el grado en que la cultura de la escuela es armónica o disruptiva respecto de la de los actores a los que atiende, lo que permitirá prever los posibles efectos y dificultades de su acción. Por último, dará a conocer los recursos que pueden pasar del medio a la escuela y la manera como «los productos» de la escuela pueden insertarse en el medio (Fernández, 2009, p. 98).

En este sentido, los actores de la comunidad que rodean a la institución educativa transfieren sus recursos - humanos, técnicos o económicos- para mejorar las condiciones de lo que ocurre dentro.

A lo largo del 2018 se llevó a cabo un proceso de relevamiento que implicó la visita a 24 escuelas ubicadas en barrios de Montevideo cuyos indicadores sociales y económicos permitían suponer que su población vive mayoritariamente en situación de vulnerabilidad social. Cabe destacar que se trataba de la totalidad de escuelas ubicadas en estos barrios. En todos los casos, se mantuvo una entrevista semiestructurada con el maestro director2 de la institución educativa o con aquella persona que la autoridad había designado para tal fin. El objetivo de las entrevistas era recabar información respecto de todas aquellas intervenciones de actores de la comunidad que habían tenido lugar en la escuela en los últimos dos años.

Este relevamiento arrojó que en las 24 escuelas visitadas habían tenido lugar 111 intervenciones llevadas adelante por diferentes actores de la comunidad. Sin embargo, un análisis más detallado del conjunto de intervenciones permite advertir algunos elementos significativos. 


\section{Dimensiones de la escuela en que impactan las intervenciones}

El primer elemento a destacar guarda relación con la dimensión de la institución educativa en la que tienen impacto las intervenciones relevadas.

En función de las entrevistas realizadas es posible afirmar que existen tres grandes dimensiones de la institución educativa en la que las intervenciones comunitarias tienen impacto: la dimensión organizacional, la dimensión administrativa y la dimensión pedagógica. Estas surgen de una reinterpretación de los aportes de Frigerio, Poggi, Tiramonti y Aguerrondo (2006) respecto de las dimensiones que se inscriben en el campo institucional.

Por dimensión organizacional se entiende el conjunto de aspectos estructurales que configuran el estilo y la forma de funcionamiento de la institución educativa. Elementos vinculados a los roles y funciones, estilos de funcionamiento, tiempos y espacios pueden ser comprendidos en el marco de esta dimensión.

Dentro de la dimensión administrativa se incluyen todos los aspectos materiales, económicos y edilicios. Es posible comprender dentro de esta aspectos vinculados al equipamiento, la mejora de espacios o el incremento de los recursos económicos para la adquisición de materiales y el desarrollo de proyectos de mejora.

Finalmente, dentro de la dimensión pedagógica es posible incluir todas aquellas acciones que se relacionan, directa o indirectamente, con la misión de la institución educativa. Esto engloba las acciones que forman parte del desarrollo curricular habitual, pero también otro conjunto de acciones más puntuales que se inscriben en proyectos específicos y que, si bien pueden tener alguna vinculación más o menos directa con elementos del currículo, se realizan con una modalidad diferente.

Entre las 111 intervenciones comunitarias reconocidas en las escuelas visitadas hay siete cuyo impacto principal tuvo que ver con la dimensión organizacional. Algunas de estas intervenciones apuntaban a la capacitación del equipo docente, mientras que otras respondían a proyectos de asesoría en la gestión institucional o a la configuración y mejora del proyecto de centro.

Otras 52 intervenciones tuvieron como objetivo central la modificación de alguna situación administrativa de las escuelas entrevistadas. Donaciones puntuales, en dinero o en especie, fueron las más frecuentes en este ámbito. Principalmente se recibieron electrodomésticos y materiales de papelería, pero también hubo donaciones puntuales de dinero. En estas intervenciones también deben destacarse los proyectos de mejora edilicia y las reparaciones puntuales que implementaron actores de la comunidad. Al respecto se ha elaborado una distinción interna que permite discriminar entre aquellas intervenciones que implican el aporte de dinero en efectivo para que la institución pueda utilizarlo (dimensión administrativa económica), aquellas que procuran mejorar los espacios físicos de la institución (dimensión administrativa edilicia) y aquellas que 
buscan la mejora de los recursos materiales, sea a través de donaciones en especie o mediante la reparación de objetos existentes en la institución (dimensión administrativa material).

Otras 52 intervenciones tuvieron como objetivo central el abordaje de la dimensión pedagógica. Proyectos puntuales sobre educación sexual, educación para la salud o educación para el cuidado del medio ambiente fueron los más frecuentes dentro de este tipo de intervenciones. En este caso se ha distinguido entre, a) intervenciones que, si bien se vinculan a los contenidos definidos por el programa escolar, no se encuentran en ningún área específica de trabajo, sino que responden a líneas transversales del sistema educativo - tales como la educación sexual, la educación en derechos humanos, la educación para la recreación y el deporte, la educación artística y otras áreas (que han sido definidas como intervenciones en la dimensión pedagógico-didáctica curricular amplia)—; b) intervenciones que apuntan directamente al abordaje de contenidos específicos dentro de un área del conocimiento que está previsto que sea tratada en el ciclo lectivo (dimensión pedagógico-didáctica curricular específica) y c) otras actividades realizadas con niñas y niños en las escuelas, que no se vinculan directamente con los contenidos curriculares previstos en la normativa vigente (dimensión pedagógicodidáctica no curricular).

En el cuadro 1 es posible observar con mayor detalle cómo se han distribuido estas intervenciones entre las dimensiones de las instituciones educativas correspondientes.

Cuadro 1. Dimensión de la institución educativa en la que impactan las intervenciones

\begin{tabular}{|cc|}
\hline Dimensión de la institución & Cantidad de intervenciones \\
\hline Organizacional & 7 \\
\hline Administrativa económica & 12 \\
\hline Administrativa edilicia & 18 \\
\hline Administrativa material & 22 \\
\hline Pedagógico-didáctica curricular amplia & 23 \\
\hline Pedagógico-didáctica curricular específica & 21 \\
\hline Pedagógico-didáctica no curricular & 8 \\
\hline Total & $\mathbf{1 1 1}$ \\
\hline
\end{tabular}

Fuente: Elaboración propia a partir de los datos recabados en el relevamiento. 


\section{Actores de la comunidad que llevan a cabo las intervenciones}

En lo que respecta a los actores a cargo de las intervenciones, un buen punto de partida es la relación entre la acción y la estructura que permite comprender su definición. De acuerdo con los planteos de Giddens:

[...] la actuación del actor (individual) tiene cuatro características definitorias: i) es una intervención intencionada, aunque sea inconsciente, ii) sobre la que el sujeto puede reflexionar y de la que es responsable, iii) que depende no tanto de las intenciones del sujeto cuanto de su capacidad y iv) que no está determinada sino que es «contingente y variable», en el sentido de que el actor tiene la posibilidad de actuar de otra manera. (Giddens citado por García Sánchez, 2007, p. 204).

Fernando Barreiro (2000) expresa que los actores sociales en el marco comunitario pueden ser comprendidos y categorizados en función del capital con que cuentan. Esto permite identificarlos como político-institucionales, territoriales o técnico-profesionales. Algunos desarrollan su tarea como resultado de una iniciativa individual. Otros lo hacen dentro de un colectivo que actúa de manera unificada, independientemente de los matices que puedan encontrarse entre sus componentes. Finalmente, otros actores desarrollan sus intervenciones en representación de una institucionalidad.

A los fines de la presente investigación se definió al actor social como cualquier persona o conjunto que desarrolla acciones determinadas en las instituciones públicas de educación formal respondiendo a su intención de intervenir en ellas, en función del lugar que ocupa en la estructura social y/o institucional en que se encuentra. Cabe destacar que los actores se ven influenciados por el contexto social, cultural o institucional en el que se desempeñan, aunque esta influencia no determine su acción.

$\mathrm{Al}$ identificar a los actores que intervienen en las escuelas relevadas, la indagación mostró que la gran mayoría de las intervenciones eran llevadas a cabo por vecinos y vecinas, organizaciones no gubernamentales (incluidas tanto asociaciones civiles y fundaciones como organizaciones comunitarias no siempre legalmente constituidas), técnicos de instituciones públicas (que de manera individual intervenían en las instituciones educativas), programas del Estado (cuyo marco de acción preveía la realización de acciones en instituciones educativas), unidades académicas y empresas.

Dentro de este marco general es posible apreciar algunos elementos llamativos que aparecen sistematizados en el cuadro 2 . 
Cuadro 2. Tipo de actor comunitario que desarrolla una intervención

\begin{tabular}{|lc|}
\hline Actor comunitario & Cantidad de intervenciones \\
\hline Unidades académicas & 17 \\
\hline Técnicos de instituciones públicas & 19 \\
Programas del Estado & 14 \\
\hline ONG & 16 \\
\hline Empresas & 19 \\
\hline Vecinos & 14 \\
\hline Acciones conjuntas o llevadas a cabo por otros actores & 12 \\
\hline Total & $\mathbf{1 1 1}$ \\
\hline
\end{tabular}

Fuente: Elaboración propia a partir de los datos recabados en el relevamiento

La mayoría de las intervenciones fueron realizadas por dos tipos de actores: las empresas y los técnicos de instituciones públicas. En ambos casos, estos actores fueron responsables de 19 intervenciones en las escuelas visitadas.

Las empresas, de manera mayoritaria, realizaron estas 19 intervenciones ofreciendo soluciones para problemas vinculados con lo económico o con carencias de infraestructura y equipamiento de la institución. Dentro de este conjunto de intervenciones se destacan las donaciones (tanto de dinero como de alimentos, electrodomésticos, materiales de papelería y oficina, recursos didácticos) y los proyectos de remodelación de espacios físicos (arreglos generales, pintura, reparación y construcción de salones).

Solamente una de las 19 intervenciones llevadas a cabo por empresas apuntó a la dimensión pedagógica, vinculando de manera directa a los trabajadores con los alumnos a través de clases de informática especializada en el rubro de la empresa en cuestión.

Los técnicos de instituciones públicas que intervinieron son en su mayoría profesionales vinculados a la Red de Atención Primaria de Salud, ${ }^{3}$ tanto médicos como psicólogos y trabajadores sociales.

De acuerdo con la definición aportada por la Organización Mundial de la Salud, la atención primaria es «la asistencia sanitaria esencial basada en métodos y tecnologías prácticos, científicamente fundados y socialmente aceptables, puesta al alcance de todos los individuos y familias de la comunidad mediante su plena participación y a un costo que la comunidad y el país puedan soportar, en todas y cada una de las etapas de su desarrollo, con un espíritu de autorresponsabilidad y autodeterminación. La atención primaria forma parte integrante tanto del sistema nacional de salud, del que constituye la función central y el núcleo principal, como del desarrollo social y económico global de la comunidad» (Vignolo, Vacarezza, Álvarez, y Sosa, 2011). En el caso de Uruguay, la Red de Atención Primaria de Salud está 
En estos casos, las intervenciones relevadas muestran una fuerte inclinación hacia el desarrollo de instancias pedagógicas, mediante talleres de educación sexual, alimentación saludable y prevención de enfermedades.

Cabe destacar que la Ley General de Educación contiene en su artículo 40 un conjunto de líneas transversales que deben ser contempladas por el sistema educativo en cualquiera de sus modalidades. Estas líneas contienen, entre otras, la educación sexual, la educación para la salud, y la educación física, la recreación y el deporte (Ley 18.347, 2009).

Otros actores de la comunidad que realizan intervenciones en las escuelas visitadas son las unidades académicas. En los últimos dos años, programas de extensión, cátedras específicas o programas de diferentes universidades y centros de educación terciaria han desarrollado algún tipo de intervención en 17 ocasiones.

Las intervenciones llevadas a cabo por las unidades académicas son sumamente diversas. En algunos casos, el relevamiento permitió identificar programas de extensión universitaria con un fuerte arraigo territorial, que intervienen en más de dos escuelas de un mismo barrio. En otros, las experiencias eran mucho más puntuales y consistían en algún proyecto específico que impactaba en una institución. Las dimensiones en las que impactaron también eran diversas. En algunos casos, se realizaron proyectos tendientes a mejorar la gestión del centro educativo (programas de formación docente, nuevas modalidades de ordenamiento escolar, colaboración con tareas administrativas y una gestión de recursos más eficiente). En otros casos se implementaron proyectos de desarrollo de un área del conocimiento (trabajando pedagógicamente desde las áreas de las ciencias humanas o naturales).

Otro escenario heterogéneo se presenta al analizar las 16 intervenciones llevadas a cabo por organizaciones no gubernamentales (ONG). Dentro de estas es posible encontrar organizaciones territoriales que desarrollan programas educativos y acciones de articulación en el marco de su tarea, así como un conjunto de fundaciones de mayor visibilidad, con alcance nacional (o, en algunos casos, internacional) y no necesariamente vinculadas geográficamente, cuya principal intervención se vincula a los aspectos económicos y materiales de las escuelas.

En cuarto lugar, encontramos 14 intervenciones realizadas por vecinos y vecinas, e igual cantidad de intervenciones desarrolladas por programas del Estado.

Los vecinos y vecinas fundamentalmente intervienen para colaborar con la mejora edilicia de las instituciones, y en algunos casos puntuales desarrollan programas específicos con una frecuencia estable (lectura de cuentos, apoyo a actividades de huerta).

configurada por un conjunto de centros de salud y policlínicas distribuidos en el territorio, lo que favorece el acceso de la población al derecho a la salud. 
En los programas del Estado también se advierte una gran diversidad. Provienen de agencias tales como el Ministerio del Interior, el Ministerio de Salud Pública, el Ministerio de Desarrollo Social, gobiernos municipales, entes autónomos (la Administración Nacional de Usinas y Transmisiones Eléctricas [UTE]) y servicios descentralizados (Obras Sanitarias del Estado [OSE]). Todas estas intervenciones implican la realización de tareas pedagógicas en función de la promoción de los derechos vinculados a la misión de la agencia que corresponda.

\section{Impacto de las intervenciones en las escuelas}

Todas las escuelas visitadas reciben al menos dos intervenciones comunitarias de alguno de los actores mencionados, y algunas registran más de 10 de actores diferentes (el máximo de intervenciones en una misma institución es de 12). Esto plantea un enorme desafío en términos de lo que implica gestionar la diversidad de actores e intervenciones comunitarias que recibe cada escuela. Más aún si se toma en consideración que, como se ha visto, las relaciones dentro de la comunidad no están exentas de tensiones y contradicciones.

\section{El desafío de gestionar la diversidad}

El conjunto de intervenciones identificadas en las escuelas entrevistadas habilita múltiples reflexiones y líneas de análisis. Una de las más relevantes en función de lo encontrado en el terreno guarda relación con la gran diversidad de intervenciones y el desafío de implementarlas de manera relativamente cohesionada en el marco de un proyecto institucional.

Como se mencionó, el relevamiento tuvo como informantes a los maestros directores de todas las escuelas ubicadas en los barrios seleccionados para la investigación, por cuanto la intención fundamental era saber cuáles eran las intervenciones realizadas, los actores comunitarios que las llevaban a cabo y la dimensión de la institución educativa en la que tenían impacto.

Si bien hasta el momento no se ha entrevistado a los actores comunitarios que llevan a cabo dichas intervenciones, es posible pensar, siguiendo los planteos desarrollados al hablar de comunidad, que las intenciones de los actores presenten tensiones y contradicciones.

¿Cuáles son los motivos, lógicas y formas de acción que implican las intervenciones llevadas a cabo por actores tan dispares? ¿Cómo conviven experiencias tan intrínsecamente diversas en la vida cotidiana de una institución educativa? Pereda (2018) da 
cuenta de las circunstancias sociales e históricas en que organizaciones sociales, fundaciones privadas y empresas, en cuanto actores de la comunidad, comienzan a realizar intervenciones en la educación. Esto revela la diversidad de miradas que dichos actores promueven e impulsan a través de sus prácticas.

Para dar respuesta a estos dos interrogantes, Hargreaves y Fink (2008) plantean que uno de los principios para la construcción de liderazgos sostenibles en el marco de los centros educativos guarda relación con la diversidad. Según estos autores, muchos de los principios que impactan en la diversidad biológica pueden ser aplicables también en el ámbito de las sociedades y grupos humanos. Entre estas características mencionan que los organismos más completos y efectivos se caracterizan por un marco de valores, objetivos y fines comunes y duraderos; la posesión y el desarrollo de la variabilidad o la diversidad en las destrezas y las facultades; los procesos que favorecen la interacción y la polinización cruzada de ideas e influencias en todo el ámbito que esa variabilidad abarca; la permeabilidad a las influencias externas; la emergencia de ideas, estructuras y procesos nuevos a medida que los elementos diversos se interconectan y otros nuevos penetran desde el exterior; la flexibilidad y adaptabilidad como respuesta al cambio medioambiental; y la elasticidad ante las amenazas y la adversidad y como reacción contra ellas (Hargreaves y Fink, 2008, p. 143).

La permeabilidad a las influencias del entorno y la posibilidad de flexibilizar y adaptar las acciones institucionales como respuesta a lo que el ambiente requiere es un elemento que enriquece el quehacer educativo y permite una mayor sostenibilidad del liderazgo, de acuerdo con los autores. Sin embargo, también mencionan la importancia de que esos procesos tengan una cohesión que impida que dichas acciones se dispersen y pierdan identidad. Esta cohesión debe encontrar su inspiración en los objetivos que encuadran el proyecto de la institución educativa.

La sostenibilidad exige diversidad [...]. La diversidad requiere menos verificaciones, mayor flexibilidad y creatividad curricular, un reconocimiento explícito y una satisfacción de las necesidades de conocimientos y aprendizaje de comunidades culturalmente diversas, del aprendizaje personalizado frente a la instrucción dictada por el correspondiente guion, la cohesión centrada en unos objetivos en vez de un alineamiento obligado y el desarrollo profesional inteligente frente a la formación del profesorado del tipo «colorear según el número» [ese juego de niños en que se pintan de un determinado color las partes de una figura según el número que lleven inscrito]. El liderazgo de organismos como sistemas naturales unidos a unos patrones modernos de comunicación interconectada nos puede ayudar a trabajar en favor, más que en contra, de la diversidad cultural de nuestros alumnos, de la diversidad profesional de nuestros profesores y de la variedad organizativa de nuestros centros (Hargreaves y Fink, 2008, p. 162). 
Esta función de dar cohesión en la diversidad es asumida por la figura del maestro director. La Legislación Escolar 23 del Consejo de Educación Inicial y Primaria, de 1961, vigente en la actualidad, dispone en su artículo 30 que parte de las funciones del maestro director de una escuela tienen que ver con «orientar y ser responsable de las relaciones de la escuela con los padres y el vecindario, así como de todas las actividades que se realicen con tales cometidos» (Consejo de Educación Inicial y Primaria [CEIP], 1961, art. 30).

Esta normativa delega en la figura del maestro director la función de coordinar las actividades que la institución educativa lleva a cabo en su vínculo con la comunidad y, por lo tanto, la responsabilidad por las intervenciones comunitarias que se realizan en cada escuela. Esto se ve complementado por el conjunto de funciones que dicha legislación otorga al maestro director en cuanto al ordenamiento y la coordinación de todas las acciones que se desarrollan en los centros educativos.

El conjunto de funciones asignadas al maestro director por la legislación vigente -en el marco de las intervenciones comunitarias en las escuelas ubicadas en contextos de vulnerabilidad social - abre una pregunta respecto de las diferentes formas de gestionar la diversidad y en qué medida se logra que dichas acciones guarden cierto nivel de cohesión institucional.

Para comprender esta tarea que atañe al rol del maestro director, surge el concepto de permeabilidad selectiva, acuñado por Jaume Trilla Bernet (1999), quien señala:

[...] decir sin más que la escuela debe arraigarse a su medio, que debe integrarse en su entorno, que debe abrirse a su contexto... constituyen, a menudo, tópicos sin matices de los que la pedagogía está demasiado llena. La función intencionalmente educativa de la escuela exige que, en su relación con el medio, lleve a cabo una tarea de selección, de criba: arraigarse pero críticamente, integrarse y abrirse según qué sí y según qué no. (Trilla Baume, 1999, p. 228)

Según el autor, este criterio de permeabilidad selectiva permite cernir para distinguir aquellas experiencias de intervención que, en función de su especificidad, intencionalidad y concordancia con el proyecto educativo que se desea alentar en términos institucionales, deben tener lugar y ser implementadas en la escuela.

La permeabilidad selectiva, pensada en los tipos de intervención señalados, implican preguntarse en qué medida las propuestas acercadas por los actores de la comunidad en materia organizacional coinciden con los proyectos de mejora que la institución desea implementar; en qué medida los aportes y colaboraciones en materia administrativa redundan en beneficios que se vinculan de manera directa con el proyecto educativo; y en qué medida las instancias pedagógicas que los actores de la comunidad proponen llevar a cabo se encuadran en un proyecto curricular en consonancia con el resto 
de la vida escolar. Mientras estas condiciones se cumplan, la intervención puede colaborar con la sostenibilidad del proyecto institucional y, lejos de convertirse en un obstáculo, realizar un aporte significativo a la vida de la escuela.

\section{Consideraciones finales}

Se ha visto el gran volumen de intervenciones comunitarias que actualmente impactan en las instituciones públicas de educación formal en contextos de vulnerabilidad social, en particular en las escuelas. Estas intervenciones son llevadas a cabo por un elenco sumamente diverso de actores de la comunidad y a su vez impactan en diferentes dimensiones de la vida institucional. Todo esto redunda en la gran heterogeneidad que se observa en el conjunto.

Esta variedad de intervenciones plantea el desafío de garantizar la cohesión institucional, confiando en los aportes que estas intervenciones pueden significar, pero asumiendo también los riesgos que implica el hecho de que se lleven adelante sin encuadrarse en un proyecto o visión institucional.

La tarea de garantizar la diversidad en la cohesión corresponde, normativamente, al maestro director. A esta figura le compete asegurar la permeabilidad selectiva en el marco de su institución, dando lugar a todas aquellas iniciativas que se articulen de manera clara con el proyecto institucional que desea alentar.

El proceso de investigación descrito continúa desarrollándose. En este marco, se han seleccionado algunas instituciones para indagar en las representaciones mutuas que existen en las intervenciones comunitarias señaladas. Con esto se quiere dar cuenta tanto de las motivaciones que llevan a los actores a intervenir en las escuelas como de las valoraciones que las instituciones educativas hacen al respecto, tanto en términos generales como sobre quien las realiza. Esta continuidad y profundización de la investigación permitirá explorar aspectos que se presentan como centrales en esta relación entre la comunidad y las instituciones educativas, y habilitar la resolución de diversos interrogantes.

En primer lugar, como se mencionaba, cabe preguntarse por las intenciones de los actores comunitarios al desarrollar sus intervenciones en las instituciones educativas. Es posible que en los relatos de empresas, organizaciones no gubernamentales, técnicos de instituciones públicas, vecinos y vecinas surjan contradicciones manifiestas en cuanto a las intenciones, y que sus concepciones de los fines de la educación se traduzcan en prácticas de intervención divergentes. A la inversa, si se expresan miradas y criterios comunes, habrá que preguntarse por qué motivos actores tan diversos coinciden en los diagnósticos y sentidos que otorgan a la educación. 
En segundo lugar, es necesario indagar qué posibilidades tienen dichos actores para incidir en el proyecto institucional de las escuelas. En la medida en que los maestros directores implementan el principio de permeabilidad selectiva en función del proyecto institucional, el proceso de construcción de este último puede develar aportes que los actores de la comunidad han hecho en su formulación.

Finalmente, cabe preguntarse si este principio de permeabilidad selectiva está siendo efectivamente implementado, es decir, en qué medida los maestros directores efectivamente ciernen, en función del proyecto institucional construido, las posibilidades de intervención de actores de comunidad que se les presentan y, en caso afirmativo, cuáles son los criterios de evaluación instrumentados para tal fin.

Independientemente de los aspectos que puedan ser profundizados en la continuidad de esta investigación, el presente relevamiento permitió conocer mejor las intervenciones comunitarias que se desarrollan en las escuelas ubicadas en contexto de vulnerabilidad social, los actores que las llevan a cabo y la dimensión de la institución educativa en la que impactan, lo que contribuye a una mejor comprensión de este tipo de experiencias.

\section{Referencias bibliográficas}

Alfieri, F. (1999). Crear cultura dentro y fuera de la escuela: algunos modelos posibles. En P. Manzano Bernández (coord.), Volver a pensar la educación (pp. 217-231). Madrid: Morata.

Alonso, N. y Viscardi, N. (2013). Gramática(s) de la convivencia. Montevideo: ANEP.

Barreiro, F. (2000). Desarrollo desde el territorio: A propósito del desarrollo local. Recuperado de http://biblioteca.municipios.unq.edu.ar/modules/mislibros/archivos/ Barreiro.pdf

Carman, M., Vieira da Cunha, N. y Segura, R. (2013). Segregación y diferencia en la ciudad. Quito: CLACSO.

Castel, R. (2009). La metamorfosis de la cuestión social: Una crónica del salariado. Buenos Aires: Paidós.

Consejo de Educación Inicial y Primaria (1961). Legislación Escolar N.ํㅜㄹ. Montevideo: CEIP.

Echeverriarza, M. P., Pereda, C. y Silveira, Y. (2011). El papel de la dirección liceal y los consejos de participación: Tensiones y sugerencias prácticas. Páginas de Educación, 4(1), 56-77. Recuperado de http://www.scielo.edu.uy/scielo.php? script= sci_arttext\&pid=S1688-74682011000100004\&lng=es\&tlng=es.

Fernández, L. (2009). Instituciones educativas. Dinámicas institucionales en situaciones críticas. Buenos Aires: Paidós. 
Frigerio, G., Poggi, M., Tiramonti, G. y Aguerrondo, I. (2006). Las instituciones educativas: cara y ceca. Elementos para su gestión. Buenos Aires: Troquel.

García Sánchez, E. (2007). El concepto de actor: Reflexiones y propuestas para la ciencia política. Andamios, 3(6), 199-216. Recuperado de http://www.scielo.org.mx/ scielo.php?script=sci_arttext\&pid=S1870-00632007000100008\&lng=es\&tlng=es.

Hargreaves, A. y Fink, D. (2008). El liderazgo sostenible: Siete principios para el liderazgo en centros educativos innovadores. Madrid: Morata.

Ley 18.437 (2009). Ley General de Educación. Montevideo: Centro de Información Oficial de la República Oriental del Uruguay.

Montero Rivas, M. (2012). El concepto de intervención social desde una perspectiva psicológico-comunitaria. Revista MEC-EDUPAZ, 1, 54-76.

Pereda, C. (2018). Organizaciones sociales, fundaciones privadas y empresas en la educación pública en Uruguay. Revista Currículo sem Fronteiras, 18(1), 185-211.

Torres, A. (2013). El retorno a la comunidad: Problemas, debates y desafíos de vivir juntos. Bogotá: CINDE.

Trilla Bernet, J. (1999). La escuela y el medio: Una reconsideración sobre el contorno de la institución escolar. En P. Manzano Bernández (coord.), Volver a pensar la educación (pp. 217-231). Madrid: Morata.

Vignolo, J., Vacarezza, M., Álvarez, C. y Sosa, A. (2011). Niveles de atención, de prevención y atención primaria de la salud. Archivos de Medicina Interna, 33(1), 7-11. Recuperado de http://www.scielo.edu.uy/scielo.php?script=sci_arttext\&pid= S1688-423X2011000100003\&lng=en\&tlng=es.

Zibechi, R. (2006). Dispersar el poder: Los movimientos como poderes antiestatales. Buenos Aires: Tinta Limón. 